\title{
Effects of yeast cell walls on performance and immune responses of cyclosporine A-treated, immunosuppressed broiler chickens
}

\author{
Shaojin Zhangt, Bingling Liaot, Xiao Li, Lei Li, Libao Ma and Xianghua Yan* \\ College of Animal Sciences and Technology, Huazhong Agricultural University, Wuban, 430070 Hubei, China
}

(Received 6 January 2011 - Revised 31 May 2011 - Accepted 2 June 2011 - First published online 26 July 2011)

\begin{abstract}
The present study was conducted to investigate the effects of dietary yeast (Saccharomyces cerevisiae) cell walls (YCW) from the yeast extract industry on performance and immune function of cyclosporine A (CSA)-treated, immunosuppressed broiler chickens. A total of 240 day-old male broilers were allocated randomly into four treatments: (1) non-challenged control; (2) non-challenged control $+0 \cdot 3 \%$ YCW; (3) CSA-challenged group; (4) CSA-challenged + 0.3\% YCW. On days 1-4 and 22-25 of age, broilers were subcutaneously injected with CSA or sterile saline. The results showed that supplementation of YCW significantly improved daily weight gain (DWG) during the starter (days 1-21, $P<0 \cdot 01$ ), finisher (days 22-42, $P<0 \cdot 01$ ) and overall (days 1-42, $P<0 \cdot 05$ ) periods compared with the control birds, but had no effect on feed conversion ratio (FCR, $P>0.05$ ). Compared with the CSA-treated birds, YCW alleviated the decrease of DWG $(P<0.01)$ and increase of FCR $(P<0.05)$ caused by CSA challenge at different periods and cumulatively. On days 21 and 42 , YCW mitigated the CSA-induced decrease of peripheral blood lymphocyte blastogenic response $(P<0 \cdot 01)$. In addition, YCW improved the relative weights of the bursa of Fabricius $(P<0 \cdot 01)$ and thymus $(P<0 \cdot 01)$ and up-regulated the splenic expression of pro-inflammatory cytokines IL-1 $\beta$ $(P<0.01)$ and IL-6 $(P<0.01)$ on day 42 compared with the CSA-treated birds. These results indicate that YCW supplementation has beneficial effects in attenuating the immunosuppressive effects of CSA challenge, therefore improving growth performance of broiler chickens.
\end{abstract}

Key words: Yeast cell walls: Cyclosporine A: Immunosuppression: Broiler chickens

In broiler production, many factors such as infectious diseases, stress and mycotoxins can result in immunosuppression ${ }^{(1)}$, which may decrease growth performance and increase disease susceptibility and mortality ${ }^{(2,3)}$, causing great economic losses ${ }^{(1,4)}$. Minimising immunosuppression and its impact is an important strategy for realising the full potential of genetic and nutritional advancements for efficient production in the broiler industry ${ }^{(4,5)}$. Despite the development of potent new antibiotics, the frequency of opportunistic infections, primarily attributed to stress-associated immunosuppression, has increased in economically important domestic food animals ${ }^{(1)}$. Moreover, the high incidence of drug-resistant bacteria from the frequent and continuous use of antibiotics poses big problems facing the poultry industry ${ }^{(1,6)}$. Thus, exploring a possible application of native immunostimulants to modulate the immune system is very important to compromised broilers, especially newly hatched broilers with immature immune function.
Saccharomyces cerevisiae is one of the most widely commercialised types of yeast, the cell wall components of which have been used in animal feeding during recent years $^{(7,8)}$. Typically, commercial yeast cell walls (YCW) are composed of $30-60 \%$ polysaccharides (15-30\% of $\beta-1,3$ / 1,6-glucan and $15-30 \%$ of mannan sugar polymers), 15-30\% proteins, 5-20\% lipids and no more than 5\% of chitin $^{(9)}$. Supplementation of broiler chicken diets with YCW or purified fractions has resulted in improvements of both body-weight gain and feed efficiency ${ }^{(10-13)}$, which has been attributed to physiological effects on the intestinal mucosa ${ }^{(11-13)}$. In addition to growth performance, YCW can also provide a widespread protection of animals against diseases, which has been primarily attributed to the stimulation of the immune system through yeast $\beta$-glucans and mannans. Numerous studies have shown that when ingested orally, yeast $\beta$-glucan can activate the cells of the immune system (macrophages), stimulate and stabilise the innate immune response in humans

\footnotetext{
Abbreviations: CSA, cyclosporine A; DFI, daily feed intake; DWG, daily weight gain; FCR, feed conversion ratio; MTT, 3-(4,5-dimethylthiazol-2-yl)-2,5diphenyltetrazolium bromide; OD, optical density; PALS, periarteriolar lymphoid sheath; RPMI-1640, Roswell Park Memorial Institute 1640; RW, relative weight; YCW, yeast cell wall.
}

*Corresponding author: X. Yan, fax +86 278728 0408, email xhyan@mail.hzau.edu.cn

$\dagger$ These authors contributed equally to the study. 
and a wide range of animals ${ }^{(14-16)}$. Exposure to $\beta$-glucan enhanced the proliferation and phagocytising efficiency of avian macrophages ${ }^{(17)}$ and heterophils ${ }^{(18)}$. Similar to mammals, broilers supplemented with $\beta$-glucans had amplified humoral $^{(17,19)}$ and cell-mediated immune responses ${ }^{(10,20)}$. Mannan oligosaccharide, another constituent of YCW, has been demonstrated to have at least three probable modes of action by which broiler performance is improved, which includes its ability to adsorb enteropathogenic bacteria, to improve gastrointestinal health and, finally, its ability to modulate the immune system ${ }^{(7,21)}$. Although many studies have been conducted to characterise the effects of YCW on the performance and immunity of broiler chickens $(8,11-13,17,19,21)$, however, most of these studies have been conducted with healthy broilers without challenge conditions. Few studies have been carried out to evaluate these effects of YCW in immunosuppressed broiler chickens.

In the present study, we used a well-documented model to induce immunosuppression in broiler chickens by injecting cyclosporine A (CSA), a potent immunosuppressive agent that has been extensively used to study the importance of cellular immunity in chickens, turkeys and pheasants ${ }^{(22)}$. The purpose of the present study was to investigate the effects of YCW obtained from $S$. cerevisiae of the yeast extract industry on the growth performance and immune function of CSA-treated, immunosuppressed broiler chickens.

\section{Experimental methods}

\section{Birds and management}

The animal protocol for the present study was approved by the Institutional Animal Care and Use Committee of the Huazhong Agricultural University (Wuhan, China). A total of 240 day-old commercial male broiler chicks (Arbor Acres, Wuhan, China) were randomly allocated into four treatments based on initial body weight $(P>0.05$, average body weight of 43.6 (SEM $0 \cdot 4) \mathrm{g}$ ). The chicks were placed in twenty-four $1 \mathrm{~m}^{2}$ wire cages with six replicate pens per treatment and ten chicks per pen. For broilers, two non-medicated maize-soyabeanbased basal diets were formulated to meet or exceed the nutrient requirements as recommended by the National Research Council (1994) during the starter (days 1-21) and finisher (days 22-42) periods (Table 1). The experimental house was provided with forced ventilation, artificial light and air conditioning. The temperature inside the house on arrival was $32^{\circ} \mathrm{C}$ and was decreased by $3^{\circ} \mathrm{C}$ each consecutive week until $20^{\circ} \mathrm{C}$ through to slaughter. The lighting programme was $23 \mathrm{~h}$ of light for the first $4 \mathrm{~d}, 20 \mathrm{~h}$ until $10 \mathrm{~d}$ and $18 \mathrm{~h}$ afterwards. Feed and water were provided for ad libitum consumption throughout the $42 \mathrm{~d}$ of experimentation.

\section{Experimental design}

Treatments included (1) non-challenged control (Control, broilers fed a control diet and injected with sterile saline), (2) non-challenged control $+0.3 \%$ YCW (YCW, broilers fed a $0.3 \%$ YCW diet and injected with sterile saline), (3) CSA-challenged group (CSA, broilers fed the same control
Table 1. Ingredient composition and calculated analysis of the starter (days 1-21) and finisher (days 22-42) basal diets for broiler chicks*

\begin{tabular}{lrr}
\hline Items & Starter & Finisher \\
\hline Ingredient & & \\
Maize (\%) & 58.80 & 63.00 \\
Soyabean meal (48\% CP) (\%) & 34.50 & 29.80 \\
Soyabean oil (\%) & 3.00 & 4.00 \\
Calcium carbonate (\%) & 0.60 & 0.50 \\
Dicalcium phosphate (\%) & 1.50 & 1.30 \\
DL-Met (99.0\%) (\%) & 0.20 & 0.10 \\
L-Lys.HCl (78.8\%) (\%) & 0.10 & - \\
Salt (\%) & 0.30 & 0.30 \\
Pre-mix feed (\%)† & 1.00 & 1.00 \\
Total (\%) & 100.00 & 100.00 \\
Calculated analysis & & \\
ME (MJ/kg) & 12.40 & 12.87 \\
CP (\%) & 20.90 & 18.99 \\
Total Lys (\%) & 1.21 & 0.99 \\
Total Met (\%) & 0.49 & 0.38 \\
Ca (\%) & 0.90 & 0.78 \\
Total P (\%) & 0.68 & 0.62 \\
Available P (\%) & 0.44 & 0.40 \\
\hline
\end{tabular}

$\mathrm{CP}$, crude protein; ME, metabolisable energy.

* The two dietary treatments were as follows: (1) the control and cyclosporine A (CSA) groups were fed the maize-soyabean-based basal diet containing $0 \%$ yeast cell walls (YCW); (2) the YCW and YCW + CSA groups were fed the basal diet into which $0.3 \%$ YCW was added at the expense of maize. (Because the cell walls are rich in carbohydrate, they replaced maize.)

† Provided per $\mathrm{kg}$ of feed: vitamin A, $5500 \mathrm{IU}(1.65 \mathrm{mg})$; vitamin $D_{3}$, $1100 \mathrm{IU}(0.0275 \mathrm{mg})$; vitamin $\mathrm{E}, 11 \mathrm{IU}(7.348 \mathrm{mg})$; vitamin $\mathrm{K}_{3}$, $1.1 \mathrm{mg}$; vitamin $B_{6}, 2.2 \mathrm{mg}$; vitamin $B_{12}, 0.0066 \mathrm{mg}$; riboflavin $4.4 \mathrm{mg}$; pantothenic acid, $11 \mathrm{mg}$; choline chloride, $220 \mathrm{mg}$; folic acid $0.55 \mathrm{mg}$; biotin, $0.11 \mathrm{mg}$; thiamin, $2.2 \mathrm{mg}$; ethoxycarbonyl quinoline, 125 mg; Mn, 120 mg; Zn, 100 mg; Fe, 60 mg; Cu, 10 mg; I, 0.46 mg.

diet and challenged with CSA); (4) CSA-challenged + 0.3\% YCW (YCW+CSA, broilers fed the same $0.3 \% \mathrm{YCW}$ diet and challenged with CSA). The dose of $0.3 \% \mathrm{YCW}$ (composed of $\beta$-glucan $\geq 20 \%$, mannan sugar polymers $\geq 15 \%$, chitin $\geq 2.5 \%$ and crude protein $\leq 35 \%$; Angel Yeast Company, Limited, Yichang, China) was chosen according to previously published studies ${ }^{(11-13)}$ and our pilot trial results. On days 1-4 and 22-25 of the trial, the CSA and YCW+CSA groups were subcutaneously injected with CSA (Sigma-Aldrich, Inc., St Louis, MO, USA) at a dosage of $50 \mathrm{mg} / \mathrm{kg}$ body weight twice daily ${ }^{(23-26)}$, and the control and YCW groups were subcutaneously injected with an equivalent amount of sterile buffered saline.

\section{Performance parameters}

Cage was the experimental unit for performance. Birds were group weighed by cage at 1, 21 and $42 \mathrm{~d}$ of age. Feed intake was monitored by cage at 21 and $42 \mathrm{~d}$ of age. Average daily weight gain (DWG), daily feed intake (DFI) and feed conversion ratio (FCR) were calculated for each period and for the overall experiment.

\section{Peripheral blood lymphocyte blastogenic assay}

To assess the cellular immune responses of the birds, a rapid colorimetric assay using 3-(4,5-dimethylthiazol-2-yl)-2,5diphenyltetrazolium bromide (MTT) was applied to evaluate 
lymphocyte blastogenic response to mitogens in the peripheral whole blood according to the method of Jaradat et al. ${ }^{(27)}$ and Kondo et al. ${ }^{(28)}$. Briefly, $2 \mathrm{ml}$ of the whole blood were collected from the brachial vein of twelve birds per treatment (two birds/ replicate pen) at 21 and $42 \mathrm{~d}$ of age (the same birds were caught and tested at 21 and $42 \mathrm{~d}$ of age unless they had died during the interval) with a heparinised syringe. Then, the blood samples were placed in sterile conical tubes and centrifuged at $50 \mathrm{~g}$ for $11 \mathrm{~min}$. The plasma and buffy coats were transferred to another tube, where the plasma was removed by centrifugation at $400 \mathrm{~g}$ for $5 \mathrm{~min}$. Cells were washed twice with T-cell medium (Roswell Park Memorial Institute (RPMI)-1640 medium (GIBCO/BRL, Eggenstein, Germany) supplemented with $2 \%$ of penicillinstreptomycin-neomycin $(5000 \mathrm{U} / \mathrm{ml}(3.125 \mathrm{mg} / \mathrm{ml}), 5 \mathrm{mg} / \mathrm{ml}$, $10 \mathrm{mg} / \mathrm{ml}$, in $0.9 \% \mathrm{NaCl}$; all from Sigma-Aldrich, Inc.)), centrifuged at $400 \mathrm{~g}$ for $5 \mathrm{~min}$, resuspended in T-cell medium and counted using the trypan blue exclusion method. Viable cells were adjusted to a concentration of $2.0 \times 10^{7} / \mathrm{ml}$ with T-cell medium.

Then, $50 \mu \mathrm{l}$ of peripheral blood lymphocytes cell suspension were added to a ninety-six-well flat-bottom microplate (PerkinElmer, Inc., Norwalk, CT, USA) containing $50 \mu \mathrm{l}$ of mitogen solution or medium (for non-stimulated cells). Plates were incubated at $40^{\circ} \mathrm{C}$ in the presence of $5 \% \mathrm{CO}_{2}$ and $95 \%$ humidity for $72 \mathrm{~h}$. Mitogen-induced stimulation was performed by phytohaemagglutinin-P (Sigma-Aldrich, Inc.). RPMI-1640 medium supplemented with $10 \mathrm{~mm}$-4-(2-hydroxyethyl)-1-piperazineethanesulfonic acid, 0.05 mm-2-mercaptoethanol, $2 \mathrm{~mm}$-L-glutamine, $1 \mathrm{~mm}$-sodium pyruvate, sodium bicarbonate $(2 \mathrm{mg} / \mathrm{ml})$, penicillin $(100 \mathrm{IU} / \mathrm{ml}$; Leica Microsystems, Solms, Germany), streptomycin $(100 \mu \mathrm{g} / \mathrm{ml})$ and $10 \%$ heat-inactivated fetal calf serum (Sigma-Aldrich, Inc.) was used as a culture medium throughout the study.

In the MTT assay, $10 \mu \mathrm{l}$ of MTT $(5 \mathrm{mg} / \mathrm{ml}$ in PBS; SigmaAldrich, Inc.) were added to each well containing cells with or without mitogen and mixed for the last $6 \mathrm{~h}$ of cultivation. At the end of the cultivation, the plates were centrifuged at $400 \mathrm{~g}$ for $10 \mathrm{~min}$ and the culture supernatant was discarded. Dimethyl sulfoxide $(100 \mu \mathrm{l})$ was added to each well, after which the plates were vigorously shaken to ensure that all crystals were completely dissolved. The amount of MTT formazan produced during the incubation was measured by an ELISA reader (Labsystems, Helsinki, Finland) at a test wavelength of $550 \mathrm{~nm}$ and a reference wavelength of $630 \mathrm{~nm}$. The results were based on the optical density (OD) at the wavelength of $550 \mathrm{~nm}\left(\mathrm{OD}_{550}\right)$ and are expressed as a stimulation index (SI), which was calculated as follows: stimulation index $=$ mean $\mathrm{OD}_{550}$ of stimulated peripheral blood lymphocytes/mean $\mathrm{OD}_{550}$ of unstimulated peripheral blood lymphocytes

\section{Relative weights of lymphoid organs}

At day 42 of age, the same twelve birds/treatment from which blood was taken were killed by cervical dislocation. The spleen, bursa of Fabricius and thymus (both right and left lobules) were excised and weighed after adherent fat from these organs was removed, and their relative weights (RW) are expressed as a percentage of live body weight.

\section{Morphology of the bursa of Fabricius and spleen}

For histological investigation, the bursa of Fabricius and spleen samples of twelve chickens from each treatment (two birds/replicate) were fixed in $10 \%$ buffered formalin for $24 \mathrm{~h}$, and after the standard histological procedure of dehydration, organ samples were embedded in paraffin. Serial cuts, $5 \mu \mathrm{m}$ in thickness, were made by microtome and stained with haematoxylin and eosin. Histological analysis was performed with a DLMS Leica light microscope connected with a camera (Leica DC-300; Leica Microsystems, Solms, Germany). Software for image analysis was IM 1000 (Leica Imaging Systems Limited, Cambridge, UK).

\section{Real-time quantitative RT-PCR}

At day 42 of age, spleen samples of twelve chickens from each treatment (two birds/replicate) were aseptically excised, rinsed in cold PBS, minced, snap-frozen in liquid $\mathrm{N}_{2}$ and stored at $-80^{\circ} \mathrm{C}$ until analysis. Total RNA was extracted from individual spleen samples using the RNAgents Total RNA Isolation System (Promega Biotech, Madison, WI, USA) following the animal tissue protocol. OD at $260 \mathrm{~nm}$ was used to determine RNA concentrations. Each total RNA sample $(2 \mu \mathrm{g})$ was reverse transcribed (Promega's Reverse Transcription System no. A3500) according to the manufacturer's instructions.

Quantitative real-time PCR analysis of spleen IL-1 $\beta$, IL- 6 and $\beta$-actin mRNA was performed using the Roche Lightcycler (Roche Diagnostics no. 2011468, Mannheim, Germany). Previously published primers for chicken IL-1 $\beta$, IL-6 and $\beta$-actin were used for $\mathrm{PCR}^{(29,30)}$, and the sequences of the primers used are listed in Table 2. PCR was performed in a reaction volume of $25 \mu \mathrm{l}$ containing the reagents at the following final concentrations: $1 \times$ SYBR Green qPCR Master Mix

Table 2. Primer sequences and annealing temperatures used for real-time quantitative RT-PCR

\begin{tabular}{|c|c|c|c|}
\hline Targets & Primer sequence $\left(5^{\prime} \rightarrow 3^{\prime}\right)$ & Annealing temperature $\left({ }^{\circ} \mathrm{C}\right)$ & GenBank accession no. \\
\hline IL-1 $\beta^{*}$ & $\begin{array}{l}\text { Forward: GCTCTACATGTCGTGTGTGATGAG } \\
\text { Reverse: TGTCGATGTCCCGCATGA }\end{array}$ & 56 & AJ245728 \\
\hline $\mathrm{IL}-6^{*}$ & $\begin{array}{l}\text { Forward: GCTCGCCGGCTTCGA } \\
\text { Reverse: GGTAGGTCTGAAAGGCGAACAG }\end{array}$ & 57 & AJ250838 \\
\hline$\beta$-Actin $†$ & $\begin{array}{l}\text { Forward: CTGACACCACACTTTCTACAATG } \\
\text { Reverse: GATCTTCATGAGGTAGTCCGTCAG }\end{array}$ & $45 \cdot 5$ & L08165 \\
\hline
\end{tabular}

* Previously published primers by Kaiser et al. ${ }^{(29)}$.

† Previously published primers by Humphrey et al. ${ }^{(30)}$. 
$(2 \times$; Roche Diagnostics Limited, Lewes, UK), forward primer $200 \mathrm{~nm}$, reverse primer $200 \mathrm{~nm}$ and $2 \mu \mathrm{l}$ of the RT product. PCR cycle conditions for all primer pairs consisted of an initial melting step at $95^{\circ} \mathrm{C}$ for $2 \mathrm{~min}$, followed by forty cycles of denaturation $\left(95^{\circ} \mathrm{C}\right.$ for $15 \mathrm{~s}$ ), annealing (annealing temperature for $15 \mathrm{~s}$; Table 2 ) and extension $\left(72^{\circ} \mathrm{C}\right.$ for $\left.45 \mathrm{~s}\right)$. After the forty cycles were completed, a melting curve analysis was performed to confirm that a single gene product was amplified, by heating samples at $65^{\circ} \mathrm{C}$ for $30 \mathrm{~s}$ and then increasing the temperature at a linear rate of $20^{\circ} \mathrm{C} / \mathrm{s}$ to $95^{\circ} \mathrm{C}$ while continuously monitoring fluorescence. Relative quantification (i.e. change in the abundance of target gene relative to untreated control) of mRNA abundance was achieved using the $2^{-\Delta \Delta C_{t}} \operatorname{method}^{(31)}$. To correct for variation in RNA preparation and sampling, $C_{\mathrm{t}}$ values for the cytokine-specific product for each sample were standardised using the $C_{\mathrm{t}}$ value of the $\beta$-actin-specific product for the same sample. Differences in cytokine expression between treatment groups were presented as fold change normalised to data from the control group chicks.

\section{Statistical analysis}

All data are presented as mean values with pooled standard errors of the mean and analysed by the one-way ANOVA procedure of the Statistical Package for Social Sciences 13.0 for Windows (SPSS, Inc., Chicago, IL, USA). Differences between means were determined using the least significant difference post hoc multiple comparison test, and the level of statistical significance was preset at $P<0.05$.

\section{Results}

\section{Growth performance}

Growth performance parameters of broiler chickens were affected by YCW and CSA during the starter, finisher and overall periods, as shown in Table 3. Compared with the control birds, YCW supplementation significantly improved DWG at different periods and cumulatively $(P<0.05)$, but DFI and FCR were not influenced at any time point during the study $(P>0.05)$, whereas the injection of CSA resulted in significantly decreased DWG $(P<0 \cdot 01)$ and increased FCR $(P<0.05)$. There were no significant differences in DWG and DFI between the YCW+CSA and control birds except for FCR. In YCW+CSA birds, FCR was not affected during the starter period; however, during the finisher and overall periods, a significantly increased FCR $(P<0 \cdot 05)$ was observed compared with the control birds. Furthermore, YCW+CSA birds had significantly increased DWG $(P<0 \cdot 01)$ and decreased FCR $(P<0.05)$ compared with the CSA birds at different periods and cumulatively.

\section{Peripheral blood lymphocyte blastogenic response}

At days 21 and 42 of age, phytohaemagglutinin-P-induced T-lymphocyte blastogenesis in the YCW-fed chicks was significantly increased compared with that in the control $(P<0.05)$, CSA $(P<0.01)$ and YCW+CSA $(P<0.05)$ groups (Table 4$)$ In contrast, the injection of CSA significantly decreased $(P<0.05)$ phytohaemagglutinin-P-induced T-lymphocyte blastogenesis compared with the control group (Table 4 ). There were no significant differences between the YCW+CSA and control birds $(P>0.05)$, but there was a significant increase $(P<0.05)$ when compared with the CSA-treated birds.

\section{Relative weights of lymphoid organs}

There was no difference in the RW of spleen among the groups; however, both the RW of the bursa of Fabricius and thymus were significantly affected by the YCW and CSA treatments (Table 5). Compared with the control birds, YCW-fed

Table 3. Effect of yeast (Saccharomyces cerevisiae) cell walls (YCW) and cyclosporin A (CSA) on the growth performance of male broiler chicks

(Mean values with their pooled standard errors, $n 60$ (ten birds/replicate) from days 1-21 and $n 48$ (eight birds/replicate) from days 22-42)

\begin{tabular}{|c|c|c|c|c|c|c|}
\hline Items* & Control & YCW & CSA & $\mathrm{YCW}+\mathrm{CSA}$ & Pooled SEM & $P$ \\
\hline \multicolumn{7}{|c|}{ DWG (g/bird per d) } \\
\hline Days $1-21$ & $35.47^{\mathrm{a}}$ & $36.45^{\mathrm{b}}$ & $34.01^{c}$ & $35.55^{\mathrm{a}}$ & 0.123 & $<0.001$ \\
\hline Days $22-42$ & $64 \cdot 25^{\mathrm{a}}$ & $67.51^{\mathrm{b}}$ & $59 \cdot 42^{\mathrm{C}}$ & $62 \cdot 32^{\mathrm{a}}$ & 0.620 & $<0.001$ \\
\hline Days 1-42 & $50 \cdot 20^{\mathrm{a}}$ & $51.95^{\mathrm{b}}$ & $46 \cdot 93^{\mathrm{c}}$ & $48 \cdot 95^{\mathrm{a}}$ & 0.339 & $<0.001$ \\
\hline \multicolumn{7}{|c|}{ DFI (g/bird per d) } \\
\hline Days 1-21 & $55 \cdot 37$ & $56 \cdot 29$ & $54 \cdot 38$ & $56 \cdot 72$ & 0.961 & 0.316 \\
\hline Days $22-42$ & $144 \cdot 25$ & $147 \cdot 39$ & 141.95 & $145 \cdot 19$ & 2.099 & 0.577 \\
\hline Days $1-42$ & $100 \cdot 79$ & $102 \cdot 31$ & $99 \cdot 14$ & $102 \cdot 60$ & 1.509 & 0.692 \\
\hline \multicolumn{7}{|l|}{ FCR $(g / g)$} \\
\hline Days $1-21$ & $1.56^{\mathrm{a}}$ & $1.52^{\mathrm{a}}$ & $1.60^{\mathrm{b}}$ & $1.58^{\mathrm{a}}$ & 0.016 & $<0.001$ \\
\hline Days $22-42$ & $2 \cdot 22^{\mathrm{a}}$ & $2 \cdot 16^{\mathrm{a}}$ & $2 \cdot 38^{\mathrm{C}}$ & $2 \cdot 29^{b}$ & 0.032 & 0.008 \\
\hline Days $1-42$ & $1.98^{\mathrm{a}}$ & $1.94^{\mathrm{a}}$ & $2.09^{C}$ & $2 \cdot 02^{b}$ & 0.023 & $<0.001$ \\
\hline
\end{tabular}

DWG, daily weight gain; DFI, daily feed intake; FCR, feed conversion ratio.

${ }_{a, b, c}$ Mean values within a row with unlike superscript letters were significantly different $(P<0.05)$.

* The four treatments were as follows: (1) non-challenged control (Control, broilers fed a control diet and injected with sterile saline); (2) non-challenged control $+0.3 \%$ YCW (YCW, broilers fed a $0.3 \%$ YCW diet and injected with sterile saline); (3) CSA-challenged group (CSA, broilers fed the same control diet and challenged with CSA); (4) CSAchallenged $+0.3 \%$ YCW (YCW + CSA, broilers fed the same $0.3 \%$ YCW diet and challenged with CSA). On days $1-4$ and 22-25 of the trial, the CSA and YCW + CSA groups were subcutaneously injected with CSA (Sigma-Aldrich, St Louis, $\mathrm{MO}$, USA) at a dosage of $50 \mathrm{mg} / \mathrm{kg}$ body weight twice daily, and the control and YCW groups were subcutaneously injected with an equivalent amount of sterile buffered saline. 
Table 4. Effect of yeast (Saccharomyces cerevisiae) cell walls (YCW) and cyclosporin A (CSA) on the blastogenic response of peripheral blood lymphocytes from 21-d-old and 42-d-old male broiler chicks

(Mean values with their pooled standard errors, $n 12$ (two birds/replicate))

\begin{tabular}{lcccccc}
\hline Item $^{*}$ & Control & YCW & CSA & YCW +CSA & Pooled SEM & $P$ \\
\hline SI† & & & & & & \\
Day 21 & $1.288^{\mathrm{a}}$ & $1.373^{\mathrm{b}}$ & $1.192^{\mathrm{c}}$ & $1.269^{\mathrm{a}}$ & 0.012 & $<0.001$ \\
Day 42 & $1.302^{\mathrm{a}}$ & $1.375^{\mathrm{b}}$ & $1.187^{\mathrm{C}}$ & $1.268^{\mathrm{a}}$ & 0.016 & $<0.001$ \\
\hline
\end{tabular}

SI, stimulation index.

${ }_{a, b, c}$ Mean values within a row with unlike superscript letters were significantly different $(P<0.05)$. The same birds were caught and tested at days 21 and 42 of age unless they had died during the interval.

* The four treatments were as follows: (1) non-challenged control (Control, broilers fed a control diet and injected with sterile saline); (2) non-challenged control + $0.3 \%$ YCW (YCW, broilers fed a $0.3 \%$ YCW diet and injected with sterile saline); (3) CSA-challenged group (CSA, broilers fed the same control diet and challenged with CSA); (4) CSA-challenged + $0.3 \%$ YCW (YCW+CSA, broilers fed the same $0.3 \%$ YCW diet and challenged with CSA). On days 1-4 and 22-25 of the trial, the CSA and YCW+CSA groups were subcutaneously injected with CSA (Sigma-Aldrich, St Louis, MO, USA) at a dosage of $50 \mathrm{mg} / \mathrm{kg}$ body weight twice daily, and the control and YCW groups were subcutaneously injected with an equivalent amount of sterile buffered saline.

† A rapid colorimetric assay using 3-(4,5-dimethylthiazol-2-yl)-2,5-diphenyltetrazolium bromide was applied to evaluate blastogenic response to mitogens of peripheral blood lymphocytes. The results were based on the optical density (OD) at the wavelength of $550 \mathrm{~nm}\left(\mathrm{OD}_{550}\right)$ and are expressed as a SI, which was calculated as follows: $\mathrm{SI}=$ mean $\mathrm{OD}_{550}$ of stimulated peripheral blood lymphocytes/mean $\mathrm{OD}_{550}$ of unstimulated peripheral blood lymphocytes.

birds had a significantly heavier bursa of Fabricius and thymus RW $(P<0 \cdot 05)$, whereas the bursa of Fabricius and thymus RW were significantly decreased $(P<0 \cdot 01)$. There were no significant differences in the bursa of Fabricius and thymus RW between the YCW+CSA and control birds $(P>0.05)$, but there was a significant increase $(P<0 \cdot 01)$ when compared with the CSA-treated birds.

\section{Morphology of the bursa of Fabricius and spleen}

Treatments did not cause significant changes in the histological structure of the bursa of Fabricius (Fig. 1). Compared with the control, the typical changes in the spleen of YCW-fed birds are reflected in the increase of lymphocytes in the splenic cords and periarteriolar lymphoid sheath (PALS), whereas spleen of CSA-treated birds was characterised by a smaller number of lymphocytes in the splenic cords and less developed of PALS (Fig. 2). There were no significant changes in the histological structure of spleen between the YCW+CSAtreated birds and the control.

\section{Splenic expression of IL-1 $\beta$ and IL-6}

At day 42, the mRNA expression of pro-inflammatory cytokines IL-1 $\beta$ and IL- 6 in the spleen was significantly upregulated in the YCW group in comparison with the control $(P<0.05)$, CSA $(P<0.01)$ and YCW+CSA $(P<0.05)$ groups (Table 6), whereas CSA-treated birds had significantly decreased expression of IL- $1 \beta$ and IL- 6 mRNA compared with the control $(P<0.01)$ and $\mathrm{YCW}+\mathrm{CSA}(P<0 \cdot 01)$ groups (Table 6). Expression of IL-1 $\beta$ and IL-6 mRNA were not significantly different between the control and YCW+CSA groups (Table 6).

\section{Discussion}

Immunosuppression is a syndrome rather than a disease and there are no 'clinical' signs. However, poor performance, uniformity problems, lower body-weight gain, increased FCR, frequent vaccine reactions, elevated mortality, common secondary bacterial infections and atrophy of lymphoid

Table 5. Effect of yeast (Saccharomyces cerevisiae) cell walls (YCW) and cyclosporin A (CSA) on the relative weights of lymphoid organs of 42-d-old male broiler chicks

(Mean values with their pooled standard errors, $n 12$ (two birds/replicate)

\begin{tabular}{llllllr}
\hline Item $^{*}$ & Control & YCW & CSA & YCW +CSA & Pooled SEM & $P$ \\
\hline $\begin{array}{l}\text { Relative weight (\% of body weight) } \\
\quad \text { Spleen }\end{array}$ & 0.12 & 0.14 & 0.12 & 0.14 & 0.017 & 0.429 \\
$\quad$ Bursa & $0.21^{\mathrm{a}}$ & $0.26^{\mathrm{b}}$ & $0.13^{\mathrm{c}}$ & $0.18^{\mathrm{a}}$ & 0.014 & $<0.001$ \\
$\quad$ Thymus & $0.48^{\mathrm{a}}$ & $0.56^{\mathrm{b}}$ & $0.38^{\mathrm{c}}$ & $0.46^{\mathrm{a}}$ & 0.019 & $<0.001$
\end{tabular}

${ }_{\mathrm{a}, \mathrm{b}, \mathrm{c}}$ Mean values within a row with unlike superscript letters were significantly different $(P<0.05)$.

* The four treatments were as follows: (1) non-challenged control (Control, broilers fed a control diet and injected with sterile saline); (2) non-challenged control + 0.3\% YCW (YCW, broilers fed a $0.3 \%$ YCW diet and injected with sterile saline); (3) CSA-challenged group (CSA, broilers fed the same control diet and challenged with CSA); (4) CSA-challenged + 0.3\% YCW (YCW + CSA, broilers fed the same $0.3 \%$ YCW diet and challenged with CSA). On days 1-4 and 22-25 of the trial, the CSA and YCW + CSA groups were subcutaneously injected with CSA (Sigma-Aldrich, St Louis, MO, USA) at a dosage of $50 \mathrm{mg} / \mathrm{kg}$ body weight twice daily, and the control and YCW groups were subcutaneously injected with an equivalent amount of sterile buffered saline. 

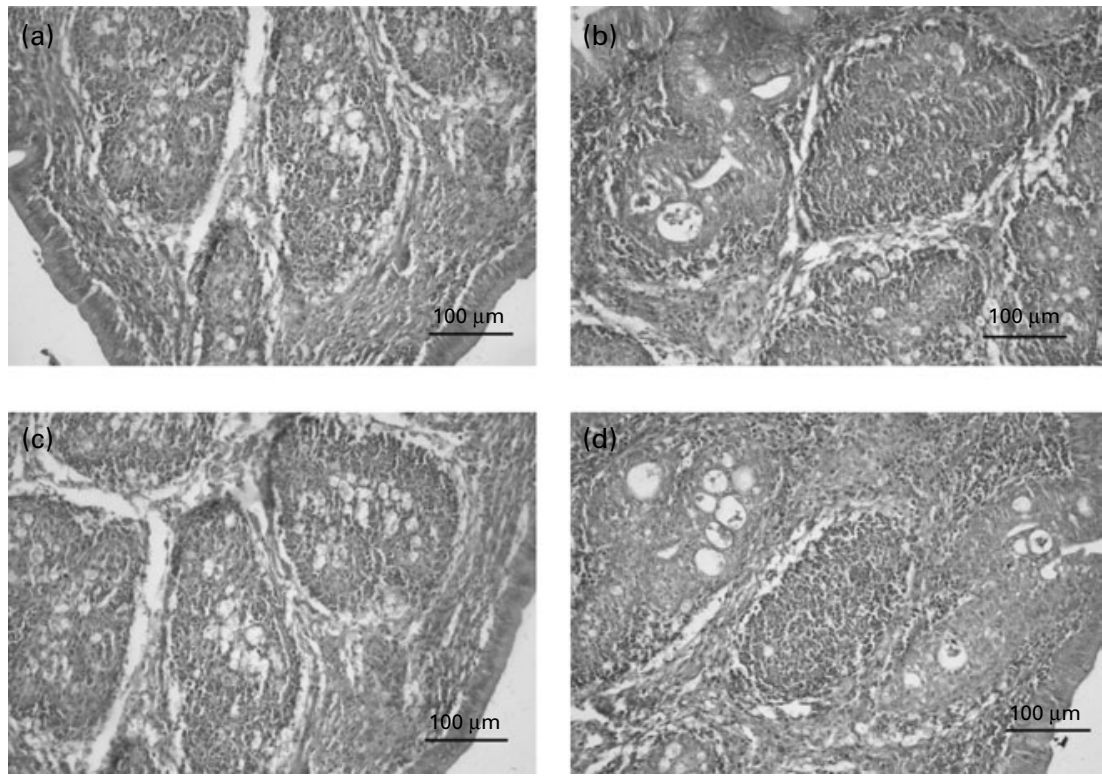

Fig. 1. Microphotographs of the chicken bursa of Fabricius $(200 \times$ magnification, haematoxylin and eosin staining). The four treatments were as follows: (1) non-challenged control ((a) Control, broilers fed a control diet and injected with sterile saline); (2) non-challenged control $+0.3 \%$ yeast cell walls (YCW) ((b) YCW, broilers fed a $0.3 \%$ YCW diet and injected with sterile saline); (3) cyclosporine A (CSA)-challenged group ((c) CSA, broilers fed the same control diet and challenged with CSA); (4) CSA-challenged + $0.3 \%$ YCW ((d) YCW +CSA, broilers fed the same $0.3 \%$ YCW diet and challenged with CSA). On days $1-4$ and 22-25 of the trial, the CSA and YCW +CSA groups were subcutaneously injected with CSA (Sigma-Aldrich, St Louis, MO, USA) at a dosage of 50 mg/kg body weight twice daily, and the control and YCW groups were subcutaneously injected with an equivalent amount of sterile buffered saline.

organs are all indicative of immunosuppression ${ }^{(1,5)}$. To evaluate whether dietary YCW supplementation could improve the immune function of immunosuppressed broiler chickens, we used a well-documented CSA-induced immunosuppressive model for broiler chickens ${ }^{(22)}$. In the present study, the CSA challenge significantly decreased the performance of broiler chickens, which is in line with the findings of Corrier et al. ${ }^{(24)}$.
Additionally, broiler chickens treated with CSA had decreased in vitro lymphocyte proliferation and lower RW of the bursa of Fabricius and thymus, which indicates that CSA suppressed immune function in broiler chickens. This outcome is consistent with reports by previous workers ${ }^{(23-26)}$, which was primarily attributed to CSA's selective and powerful inhibiting effects on the early phase of T-lymphocyte activation ${ }^{(32)}$.
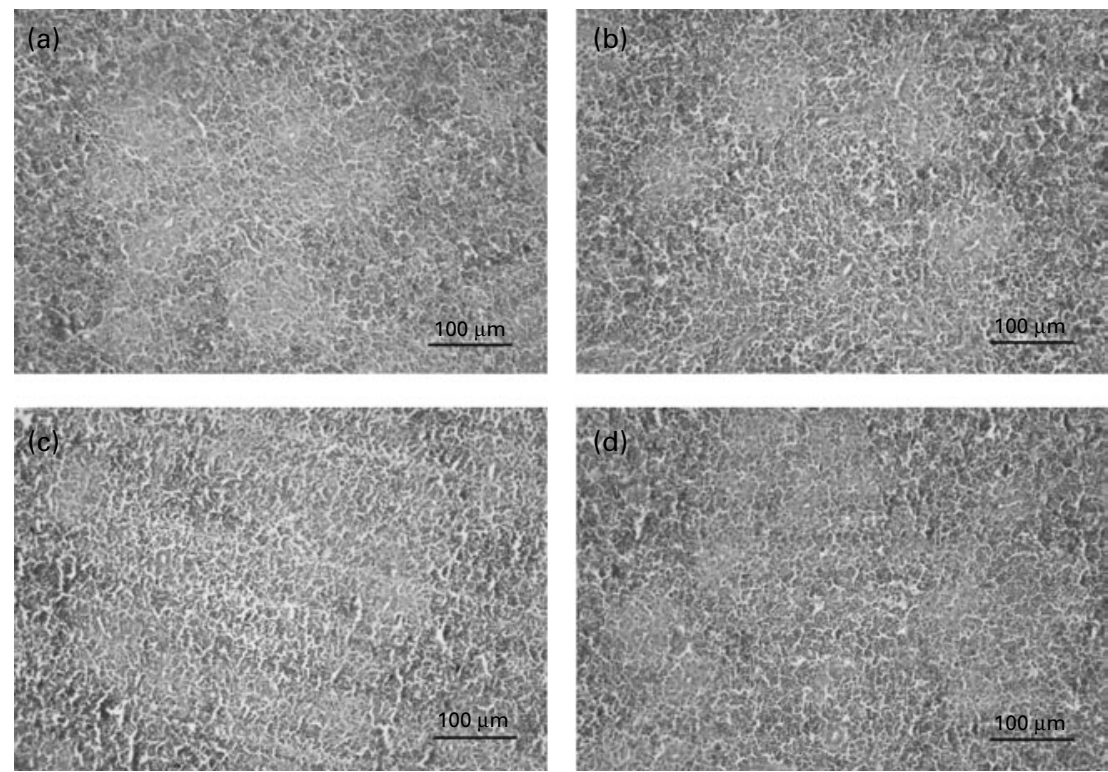

Fig. 2. Microphotographs of chicken spleen $(200 \times$ magnification, haematoxylin and eosin staining). The four treatments were as follows: (1) non-challenged control ((a) Control, broilers fed a control diet and injected with sterile saline); (2) non-challenged control $+0.3 \%$ yeast cell walls (YCW) ((b) YCW, broilers fed a $0.3 \%$ YCW diet and injected with sterile saline); (3) cyclosporine A (CSA)-challenged group ((c) CSA, broilers fed the same control diet and challenged with CSA); (4) CSA-challenged + 0.3\% YCW ((d) YCW + CSA, broilers fed the same $0.3 \%$ YCW diet and challenged with CSA). On days 1-4 and $22-25$ of the trial, the CSA and YCW+CSA groups were subcutaneously injected with CSA (Sigma-Aldrich, St Louis, MO, USA) at a dosage of $50 \mathrm{mg} / \mathrm{kg}$ body weight twice daily, and the control and YCW groups were subcutaneously injected with an equivalent amount of sterile buffered saline. 
Table 6. Effect of yeast (Saccharomyces cerevisiae) cell walls (YCW) and cyclosporin A (CSA) on the relative expression of IL-1 $\beta$ and IL- 6 in the spleen of 42 -d-old male broiler chicks (Mean values with their pooled standard errors, $n 12$ (two birds/replicate))

\begin{tabular}{lcccccc}
\hline Item $^{*}$ & Control & YCW & CSA & YCW +CSA & Pooled SEM & $P$ \\
\hline Relative expression levels (\%)† & & & & & & \\
IL-1 $\beta$ & $1.00^{\mathrm{a}}$ & $1.61^{\mathrm{b}}$ & $0.56^{\mathrm{c}}$ & $1.12^{\mathrm{a}}$ & 0.095 & $<0.001$ \\
IL-6 & $1.00^{\mathrm{a}}$ & $1.48^{\mathrm{b}}$ & $0.46^{\mathrm{c}}$ & $1.16^{\mathrm{a}}$ & 0.066 & $<0.001$
\end{tabular}

a,b,c Mean values within a row with unlike superscript letters were significantly different $(P<0.05)$.

* The four treatments were as follows: (1) non-challenged control (Control, broilers fed a control diet and injected with sterile saline); (2) non-challenged control $+0.3 \%$ YCW (YCW, broilers fed a $0.3 \%$ YCW diet and injected with sterile saline); (3) CSAchallenged group (CSA, broilers fed the same control diet and challenged with CSA); (4) CSA-challenged + 0.3\% YCW (YCW+ CSA, broilers fed the same $0.3 \%$ YCW diet and challenged with CSA). On days 1-4 and 22-25 of the trial, the CSA and YCW + CSA groups were subcutaneously injected with CSA (Sigma-Aldrich, St Louis, MO, USA) at a dosage of $50 \mathrm{mg} / \mathrm{kg}$ body weight twice daily, and the control and YCW groups were subcutaneously injected with an equivalent amount of sterile buffered saline.

$\dagger$ Relative gene expression was calculated using the $2^{-\Delta \Delta C_{t}}$ method with $\beta$-actin as the endogenous control and the average $\Delta C_{t}$ value for the control of the $0.0 \% \mathrm{YCW}$-fed birds on day 42 as the calibrator.

The worldwide trend towards eliminating antibiotic growth promoters in balanced feed has led to a search for alternatives that provide benefits similar to antibiotic growth promoters. The present results showed that dietary YCW supplementation significantly improved DWG compared with the control birds. Similar results with regard to the effect of YCW supplementation on performance have been reported in poultry ${ }^{(11-13)}$. Santin et $a l .{ }^{(11)}$ reported that broilers receiving feed containing $0 \cdot 2 \%$ YCW showed higher DWG and better FCR. Zhang et $a{ }^{(12)}$ reported that $0.3 \%$ YCW-fed birds had higher DWG and lower FCR. Morales-Lopez et al. ${ }^{(13)}$ reported that $500 \mathrm{mg} / \mathrm{kg}$ of yeast extract-YCW increased body weight and DFI ( $42 \mathrm{~d}$ ) on a wheat-based diet, whereas $500 \mathrm{mg} / \mathrm{kg}$ of yeast extract-YCW and $500 \mathrm{mg} / \mathrm{kg}$ of brewery-YCW treatments both improved feed efficiency with respect to the negative control group (1-14 d) on a maize-based diet. Increased villus height ${ }^{(11-13)}$, mucosa thickness ${ }^{(11-13)}$ and number of goblet cells ${ }^{(13)}$ were observed in YCW-fed broilers of these experiments, suggesting that supplementation of $\mathrm{YCW}$ to broiler chicken diets increased animal performance by favouring intestinal mucosa development. However, DFI and FCR were not affected by YCW supplementation compared with the control birds in the present study. The variable results of these studies could be due to a variety of reasons such as differences in the source and preparation of the YCW or the presence and type of challenge utilised, which may influence the responses observed. In addition, YCW+CSA birds had significantly increased DWG and decreased FCR at different periods and cumulatively compared with CSA birds, which suggests that YCW attenuated the growth-suppressive effects of the CSA treatment. Considering that CSA challenge significantly decreased the performance of broiler chickens via suppressing the immune system; therefore, it is possible that $0.3 \% \mathrm{YCW}$ supplementation attenuated the growth-suppressive effects of the CSA challenge by enhancing the immune function of the birds.

The mitogen-induced blastogenic response assay of peripheral blood lymphocytes has been widely used to assess non-specific cellular immunity ${ }^{(28,33)}$. In the present study, dietary supplementation of $0.3 \% \mathrm{YCW}$ enhanced lymphocyte proliferation compared with the control birds and mitigated the decrease of lymphocyte proliferation caused by CSA challenge. The present findings are consistent with the results of Gomez-Verduzco et al. ${ }^{(34)}$ who reported that dietary supplementation of $0.05 \%$ of YCW increased humoral and cell-mediated immune responses. Verduzco et al. ${ }^{(35)}$ also reported that addition of YCW increased cellular immune response as documented by the basophilic cutaneous hypersensitivity test. This may result from the chemical structure of YCW, which consists mainly of sugars that function as a lectin-type receptor ligand ${ }^{(35)}$.

Lymphoid organ weights are easily measured and reflect the body's ability to provide lymphoid cells during an immune response. Classically, immunosuppressed birds have smaller lymphoid organs ${ }^{(36)}$. In the present study, $0.3 \%$ YCW supplementation increased the RW of the bursa of Fabricius and thymus on day 42 compared with the control birds, whereas YCW+CSA birds had significantly heavier RW of both these two organs compared with the CSA birds. In agreement with the present findings, Morales-Lopez et al. ${ }^{(8)}$ observed a tendency of heavier RW of lymphoid organs in broilers fed $500 \mathrm{mg} / \mathrm{kg}$ of YCW. Guo et al. ${ }^{(17)}$ and Zhang et al. ${ }^{(19)}$ observed an increase in the spleen, bursa of Fabricius and thymus RW of broilers fed diets supplemented with 40 or $50 \mathrm{mg} \beta$-glucan $/ \mathrm{kg}$ of feed. Corrier et al. ${ }^{(24)}$ also found that chicks subcutaneously injected with CSA at a dosage of $50 \mathrm{mg} / \mathrm{kg}$ body weight twice daily for the first $4 \mathrm{~d}$ after hatching had a significantly decreased bursa of Fabricius RW compared with the control group at $10 \mathrm{~d}$ of age, and spleen RW was not affected by the CSA treatment. According to Ferket et al. ${ }^{(37)}$, the YCW components might stimulate the gut-associated immune system by acting as a non-pathogenic microbial antigen, giving an adjuvant-like effect. Studies in mammals have described the importance of digestive microbial antigen stimulation on the development of lymphoid organ tissue ${ }^{(38,39)}$. Therefore, it is possible that YCW supplementation improved the RW of lymphoid organs and attenuated the suppressive effects of the CSA challenge by stimulating the gut-associated immune system.

Histological analysis of broiler chickens' immune organs showed significant differences in the structure of spleen in different groups. Considering the role of the spleen in immunogenesis, special attention was paid to the structure of the splenic cords and PALS during histological examination. 
In the present study, there was an increase in the number of lymphocytes in the splenic cords and better-developed PALS in the spleen of YCW-fed birds compared with control birds, whereas similar results were observed in the spleen of YCW+CSA-treated birds compared with CSA-treated birds, suggesting that YCW can exert beneficial effects on the spleen of not only healthy but also CSA-treated, immunosuppressed broiler chickens. The present findings are consistent with the results of Nowak et al. ${ }^{(26)}$ who reported that the major histological changes of chicks receiving a CSA dose of $50 \mathrm{mg} / \mathrm{kg}$ by intramuscular administration of the drug in a neutral oil every $3 \mathrm{~d}$ were in the spleen where there was a depletion of the PALS, a marked increase in granulated cells, a decrease in germinal centres and the appearance of large numbers of Mott cells.

In chickens, the spleen acts both as a reservoir and activation site for leucocytes, therefore splenic gene expression reflects systemic immune function ${ }^{(40)}$. To future explore the effect of $\mathrm{YCW}$ on modulating immune function in immunosuppressed broiler chickens, the splenic expression of IL- $1 \beta$ and IL- 6 was measured in the present study. IL- $1 \beta$ and IL-6 are pro-inflammatory cytokines that are primarily produced by activated phagocytes, especially macrophages, as well as endothelial cells ${ }^{(41,42)}$. They are particularly important in initiating an acute-phase immune response, recruiting and activating leucocytes and setting the stage for appropriate action in response to microbes, tissue injury and other components that signal danger ${ }^{(41-44)}$. Hence, it is not surprising that the expression of these cytokines was significantly down-regulated in the spleen of CSA-treated birds, as it has been reported that CSA prevents the synthesis of cytokines by T-cells by blocking a late-stage signalling pathway initiated by the T-cell receptor, which affects the production of IL- 2 at the transcript level ${ }^{(45-48)}$. We found that IL-1 $\beta$ and IL-6 expressions were significantly enhanced in the spleen of YCW-fed birds, suggesting that YCW might act as an immunoprotective agent by up-regulating the inflammatory response leading to enhanced protection against pathogens. Furthermore, YCW+CSA significantly up-regulated the splenic expression of IL-1 $\beta$ and IL-6 compared with the CSA-treated birds, indicating that YCW are still effective in up-regulating the inflammatory response in CSA-treated, immunosuppressed birds. These findings suggest that YCW may enhance the cellmediated immune response in broiler chickens by modulating the production of cytokines.

In conclusion, these data suggest that dietary supplementation of YCW exerts beneficial effects in attenuating the immunosuppressive effects of CSA challenge, therefore improving the growth performance of broiler chickens.

\section{Acknowledgements}

The authors have declared that no conflict of interest exists. The present study was supported by grants from the National Natural Science Foundation of China (no. 31072036 and 30700580) and the Huazhong Agricultural University Scientific \& Technological Self-innovation Foundation (no. 2010PY011). X. Y., L. M. and B. L. designed the research; S. Z. and B. L. performed the research; S. Z., X. L., L. L. and X. Y. analysed the data; S. Z., X. L. and X. Y. wrote the manuscript.

\section{References}

1. El-Abasy M, Motobu M, Nakamura K, et al. (2004) Preventive and therapeutic effects of sugar cane extract on cyclophosphamide-induced immunosuppression in chickens. Int Immunopharmacol 4, 983-990.

2. He X, Yang X \& Guo Y (2007) Effects of different dietary oil sources on immune function in cyclophosphamide immunosuppressed chickens. Anim Feed Sci Technol 139, 186-200.

3. Tayade C, Jaiswal TN, Mishra SC, et al. (2006) L-Arginine stimulates immune response in chickens immunized with intermediate plus strain of infectious bursal disease vaccine. Vaccine 24, 552-560.

4. Fussell LW (1998) Poultry industry strategies for control of immunosuppressive diseases. Poult Sci 77, 1193-1196.

5. Hoerr FJ (2010) Clinical aspects of immunosuppression in poultry. Avian Dis 54, 2-15.

6. Huyghebaert G, Ducatelle R \& Van Immerseel F (2011) An update on alternatives to antimicrobial growth promoters for broilers. Vet $J \mathbf{1 8 7}, 182-188$.

7. Hooge DM (2004) Meta-analysis of broiler chicken pen trials evaluating dietary mannan oligosaccharide, 1993-2003. Int J Poult Sci 3, 163-174.

8. Morales-Lopez R, Auclair E, Garcia F, et al. (2009) Use of yeast cell walls; $\beta-1,3 / 1$, 6-glucans; and mannoproteins in broiler chicken diets. Poult Sci 88, 601-607.

9. Aguilar-Uscanga B \& Francois JM (2003) A study of the yeast cell wall composition and structure in response to growth conditions and mode of cultivation. Lett Appl Microbiol 37, 268-274.

10. Chae BJ, Lohakare JD, Moon WK, et al. (2006) Effects of supplementation of beta-glucan on the growth performance and immunity in broilers. Res Vet Sci 80, 291-298.

11. Santin E, Maiorka A, Macari M, et al. (2001) Performance and intestinal mucosa development of broiler chickens fed diets containing Saccharomyces cerevisiae cell wall. J Appl Poult Res 10, 236-244.

12. Zhang AW, Lee BD, Lee SK, et al. (2005) Effects of yeast (Saccharomyces cerevisiae) cell components on growth performance, meat quality, and ileal mucosa development of broiler chicks. Poult Sci 84, 1015-1021.

13. Morales-Lopez R, Auclair E, Van Immerseel F, et al. (2010) Effects of different yeast cell wall supplements added to maize- or wheat-based diets for broiler chickens. Br Poult Sci 51, 399-408.

14. Abel G \& Czop JK (1992) Stimulation of human monocyte [beta]-glucan receptors by glucan particles induces production of TNF-[alpha] and IL-1[beta]. Int J Immunopharmacol 14, 1363-1373.

15. Cleary JA, Kelly GE \& Husband AJ (1999) The effect of molecular weight and beta-1,6-linkages on priming of macrophage function in mice by $(1,3)$-beta-D-glucan. Immunol Cell Biol 77, 395-403.

16. Cross GG, Jennings HJ, Whitfield DM, et al. (2001) Immunostimulant oxidized beta-glucan conjugates. Int Immunopharmacol 1, 539-550.

17. Guo YM, Ali RA \& Qureshi MA (2003) The influence of beta-glucan on immune responses in broiler chicks. Immunopharmacol Immunotoxicol 25, 461-472.

18. Lowry VK, Farnell MB, Ferro PJ, et al. (2005) Purified beta-glucan as an abiotic feed additive up-regulates the 
innate immune response in immature chickens against Salmonella enterica serovar Enteritidis. Int J Food Microbiol 98, 309-318.

19. Zhang B, Guo YM \& Wang Z (2008) The modulating effect of beta-1,3/1,6-glucan supplementation in the diet on performance and immunological responses of broiler chickens. Asian Austral J Anim 21, 237-244.

20. Chen HL, Li DF, Chang BY, et al. (2003) Effects of lentinan on broiler splenocyte proliferation, interleukin-2 production, and signal transduction. Poult Sci 82, 760-766.

21. Shashidhara RG \& Devegowda G (2003) Effect of dietary mannan oligosaccharide on broiler breeder production traits and immunity. Poult Sci 82, 1319-1325.

22. Rubbenstroth D, Dalgaard TS, Kothlow S, et al. (2010) Effects of cyclosporin A induced T-lymphocyte depletion on the course of avian metapneumovirus (aMPV) infection in turkeys. Dev Comp Immunol 34, 518-529.

23. Poonia B \& Charan S (2001) T-cell suppression by cyclosporin-A enhances infectious bursal disease virus infection in experimentally infected chickens. Avian Pathol 30, 311-319.

24. Corrier DE, Elissalde MH, Ziprin RL, et al. (1991) Effect of immunosuppression with cyclophosphamide, cyclosporin, or dexamethasone on Salmonella colonization of broiler chicks. Avian Dis 35, 40-45.

25. Lillehoj HS (1987) Effects of immunosuppression on avian coccidiosis: cyclosporin A but not hormonal bursectomy abrogates host protective immunity. Infect Immunol $\mathbf{5 5}$, $1616-1621$.

26. Nowak JS, Kai O, Peck R, et al. (1982) The effects of cyclosporin A on the chicken immune system. Eur J Immunol 12, $867-876$

27. Jaradat ZW, Vila B \& Marquardt RR (2006) Adverse effects of T-2 toxin on chicken lymphocytes blastogenesis and its protection with vitamin E. Toxicology 225, 90-96.

28. Kondo T, Sugiura T, Kamada M, et al. (1996) Colorimetric assay of equine peripheral lymphocyte blastogenesis using MTT. J Equine Sci 7, 63-66.

29. Kaiser P, Underwood G \& Davison F (2003) Differential cytokine responses following Marek's disease virus infection of chickens differing in resistance to Marek's disease. $J$ Virol 77, $762-768$.

30. Humphrey BD, Stephensen CB, Calvert CC, et al. (2004) Glucose and cationic amino acid transporter expression in growing chickens (Gallus gallus domesticus). Comp Biochem Physiol A Mol Integr Physiol 138, 515-525.

31. Livak KJ \& Schmittgen TD (2001) Analysis of relative gene expression data using real-time quantitative PCR and the 2(T)(-Delta Delta C) method. Methods 25, 402-408

32. Tocci MJ \& Sigal NH (1992) Recent advances in the mechanism of action of cyclosporine and FK506. Curr Opin Nephrol Hypertens 1, 236-242.
33. Heckert RA, Estevez I, Russek-Cohen E, et al. (2002) Effects of density and perch availability on the immune status of broilers. Poult Sci 81, 451-457.

34. Gomez-Verduzco G, Cortes-Cuevas A, Lopez-Coello C, et al. (2009) Dietary supplementation of mannan-oligosaccharide enhances neonatal immune responses in chickens during natural exposure to Eimeria spp. Acta Vet Scand 51, 11.

35. Verduzco GG, Cuevas AC, Coello CL, et al. (2009) Productive performance and immune response in broilers fed a sorghum plus soy diet supplemented with yeast (Saccharomyces cerevisiae) cell walls, in the presence or absence of aflotoxin B1. Tecnica Pecuaria En Mexico 47, 285-297.

36. Pope CR (1991) Pathology of lymphoid organs with emphasis on immunosuppression. Vet Immunol Immunopathol 30, 31-44.

37. Ferket PR, Parks CW \& Grimes JL (2002) Benefits of dietary antibiotic and mannan oligosaccharide supplementation for poultry. Proceedings of the Multi-State Poultry Feeding and Nutrition Conference, Indianapolis, IN 5-24.

38. Pabst R, Geist M, Rothkotter HJ, et al. (1988) Postnatal development and lymphocyte production of jejunal and ileal Peyer's patches in normal and gnotobiotic pigs. Immunology 64, 539-544.

39. Rothkotter HJ, Ulbrich H \& Pabst R (1991) The postnatal development of gut lamina propria lymphocytes: number, proliferation, and $\mathrm{T}$ and $\mathrm{B}$ cell subsets in conventional and germ-free pigs. Pediatr Res 29, 237-242.

40. Redmond SB, Tell RM, Coble D, et al. (2010) Differential splenic cytokine responses to dietary immune modulation by diverse chicken lines. Poult Sci 89, 1635-1641.

41. Van Snick J (1990) Interleukin-6: an overview. Annu Rev Immunol 8, 253-278.

42. Dinarello CA (2005) Interleukin-1[beta]. Crit Care Med 33, S460-S462.

43. Hamal KR, Wideman RF, Anthony NB, et al. (2010) Differential gene expression of proinflammatory chemokines and cytokines in lungs of ascites-resistant and -susceptible broiler chickens following intravenous cellulose microparticle injection. Vet Immunol Immunopathol 133, 250-255.

44. Wigley P \& Kaiser P (2003) Avian cytokines in health and disease. Rev Bras Cienc Avic 5, 1-14.

45. Hill JE, Rowland GN, Latimer KS, et al. (1989) Effects of cyclosporine A on reovirus-infected broilers. Avian Dis 33, $86-92$.

46. Wiederrecht G, Lam E, Hung S, et al. (1993) The mechanism of action of FK-506 and cyclosporin A. Ann N Y Acad Sci 696, 9-19.

47. Zenke G, Baumann G, Wenger R, et al. (1993) Molecular mechanisms of immunosuppression by cyclosporins. Ann N Y Acad Sci 685, 330-335.

48. Resch K \& Szamel M (1997) Molecular mechanisms of the immunosuppressive action of cyclosporin A. Int $J$ Immunopharmacol 19, 579-585. 